\title{
Nanoscale Pattern Formation during Electrodeposition: Ru on Reconstructed Au(111)
}

\author{
S. Strbac,* O. M. Magnussen, and R. J. Behm \\ Abteilung Oberflächenchemie und Katalyse, Universität Ulm, D-89069 Ulm, Germany
}

(Received 15 January 1999)

\begin{abstract}
Scanning tunneling microscopy results on $\mathrm{Ru}$ electrodeposition on reconstructed $\mathrm{Au}(111)$ are presented, which show a novel type of site-selective nucleation on this surface. At potentials around $0.0 \mathrm{~V}$ vs the $\mathrm{Ag} / \mathrm{AgCl}$ reference electrode, nucleation of $\mathrm{Ru}$ monolayer islands proceeds almost exclusively in the fcc regions of the reconstructed surface rather than at the elbow sites as commonly found for metal/Au(111) systems. These results provide a link between nucleation on two-dimensional and uniaxial dislocation networks. The nucleation behavior allows the formation of well-defined nmscale admetal structures which replicate the reconstruction pattern.
\end{abstract}

PACS numbers: 68.55.-a, 61.16.Ch, 81.15.Pq

Growth on patterned substrates, where the surface exhibits ordered inhomogeneities on the nanometer scale, has recently attracted considerable interest since it offers unique opportunities for the preparation of equally spaced and sized admetal structures [1-4]. An important class of such patterned surfaces are ultrathin heteroepitaxial films, where strain relief in the admetal layer or film often causes the formation of periodic surface dislocation networks with typical spacings of a few nm and one- or two-dimensional periodicity $[4,5]$. In the presence of dislocation networks the binding energies and the energy barriers for adatom diffusion are periodically modulated, which can strongly influence the nucleation and growth behavior on these patterned surfaces $[1,3,4,6]$. Choosing suitable growth parameters, nucleation can be restricted to preferred sites on the heterogeneous surface; i.e., the dislocation networks act as nm-scale templates for the formation of periodic admetal structures.

A well-known example is the herringbone reconstructed $\mathrm{Au}(111)$ surface, where surface dislocations separate alternating regions of fcc and hcp stacking in the topmost Au layer [7]. Here a characteristic, selective nucleation of adislands at specific defect sites of the reconstructed surface, the bending points of the dislocation domains, was observed for Ni and other metals $[1,6]$. Since the same dislocation structure is found also on $\mathrm{Au}(111)$ electrodes immersed in electrolyte solutions at potentials negative of a critical potential, which is determined by the anion species in the electrolyte $(0.25 \mathrm{~V}$ vs the $\mathrm{Ag} / \mathrm{AgCl}$ reference electrode in $0.5 \mathrm{M} \mathrm{H}_{2} \mathrm{SO}_{4}$ ) [8-11], similar schemes for nanoscale pattern formation are expected also for electrodeposition. Indeed, a similar nucleation behavior was reported for Ni electrodeposition on reconstructed $\mathrm{Au}(111)$ at low overpotentials [2].

So far, however, the ordered deposit patterns obtained on epitaxial film templates with two-dimensional dislocation networks [3,4] differ from those observed on the uniaxial dislocation network on the reconstructed $\mathrm{Au}(111)$ substrate $[1,2,6]$. In the latter case the preferential nucleation sites were at the elbows of the dislocation lines, both for gas phase deposition or for electrodeposition, while in the former case, which up to now was observed only for gas-phase deposition, nucleation took place between the dislocation lines, in the fcc-stacked areas within the dislocation triangle. This leads to the question whether there are principal differences between nucleation on one- and on two-dimensional dislocation networks or whether these differences are coincidental. Here we present in situ scanning tunneling microscopy (STM) results for $\mathrm{Ru}$ electrodeposition on reconstructed $\mathrm{Au}(111)$ electrodes which decide this question in the latter sense, showing that (i) the nucleation behavior for a given template depends on the nature of the deposit (and the deposition parameters) and that (ii) for suitable deposits preferential nucleation between the dislocation lines is possible also on the reconstructed $\mathrm{Au}(111)$ substrate, i.e., on a uniaxial dislocation network. The data reveal a new type of growth on this heterogeneous substrate, namely, the (potential-dependent) selective nucleation of admetal islands in the fcc areas within the reconstruction unit cell, and the subsequent coalescence of these islands into nanowires along the fcc domains. This behavior results in a 1:1 Ru replica of the original dislocation pattern.

The experiments were performed using a homebuilt STM similar to that described in Ref. [12]. Electrochemically etched Pt-Ir or W tips coated with Apiezon wax were used. Tip and sample potentials were independently kept under potential control and measured versus a $\mathrm{Ag} / \mathrm{AgCl}$ ( $\mathrm{KCl}$ saturated) reference electrode. Since the $\mathrm{Ru}$-containing electrolyte is not stable, i.e., the $\mathrm{RuCl}_{3}$ is slowly converted into $\mathrm{RuO}\left[\left(\mathrm{H}_{2} \mathrm{O}\right)_{4}\right]^{2+}$, the solution was freshly prepared for each measurement from $\mathrm{RuCl}_{3} \cdot \mathrm{aq}$ (Fluka), suprapure $\mathrm{H}_{2} \mathrm{SO}_{4}$ (Merck), and Milli-Q water. The STM experiments were performed on a flame annealed $\mathrm{Au}(111)$ single crystal employing two different procedures. In the first one the deposition process was observed directly by STM using Pt-Ir tips and tip potentials of 0.4 to $0.5 \mathrm{~V}$ to avoid $\mathrm{Ru}$ deposition on the tunneling tip. Although this allowed us to observe the initial stages of $\mathrm{Ru}$ deposition (see below), deposition of 
$\mathrm{Ru}$ was limited to coverages $<0.01 \mathrm{ML}$ in these experiments due to pronounced tip shielding effects. Hence, in a second procedure $\mathrm{Ru}$ was deposited by immersing the $\mathrm{Au}$ sample at the deposition potential into Ru-containing electrolyte, which was subsequently replaced with pure, $\mathrm{Ru}-$ free $0.5 \mathrm{M} \mathrm{H}_{2} \mathrm{SO}_{4}$ solution by exchanging the solution for $10-15 \mathrm{~s}(\approx 10 \times$ cell volume $)$ at the same potential [13]. Finally the surface morphology was characterized by in situ STM. Ru coverages refer to the fraction of covered $\mathrm{Au}$ surface area and were determined directly from the STM images (relative error 5\%). These were obtained in constant current mode with tunneling currents between 1-10 nA and are presented as top view images with darker colors corresponding to lower surface areas.

Cyclic voltammetry measurements of $\mathrm{Ru}$ electrodeposition on $\mathrm{Au}(111)$ show an enhanced double layer current and a continuously increasing cathodic current negative of $0.3 \mathrm{~V}$. Hence, $\mathrm{Ru}$ deposition takes place not only at potentials close to the $\mathrm{Ru}^{2+} / \mathrm{Ru}^{0}$ equilibrium potential $\left(E_{\mathrm{Ru}^{2+} / \mathrm{Ru}^{0}}=0.135 \mathrm{~V}\right.$ for $10^{-4} \mathrm{M} \mathrm{RuCl} 3$ solution), but, to a lesser degree, also in the entire double layer potential regime up to the onset of $\mathrm{Au}$ oxidation [13]. A similar behavior was reported also for $\mathrm{Ru}$ electrodeposition on $\operatorname{Pt}(111)$ electrodes and attributed to the reduction of $\mathrm{RuO}\left[\left(\mathrm{H}_{2} \mathrm{O}\right)_{4}\right]^{2+}$ species to metallic $\mathrm{Ru}$ or to $\mathrm{RuOH}$ [14]. Therefore STM measurements were predominantly performed in Ru-free electrolyte to avoid continuing deposition. The deposition potential was generally chosen negative of $E_{\mathrm{Ru}^{2+} / \mathrm{Ru}^{0}}$. The deposited $\mathrm{Ru}$ is irreversibly bound to the Au surface and can be dissolved only at potentials in the Au oxidation range.

In the STM experiments the topography of the reconstructed $\mathrm{Au}(111)$ surface was characterized first in Ru-free $0.5 \mathrm{M} \mathrm{H}_{2} \mathrm{SO}_{4}$ solution. Because of experimental reasons the initial surface exhibits extended domains of unidirectional double rows of the reconstruction [15] (see Fig. 1) rather than the periodic zigzag pattern of the herringbone structure. The initial stages of Ru electrodeposition are illustrated in Fig. 1, which was recorded in the Ru containing electrolyte after decreasing the potential from values positive of $E_{\mathrm{Ru}^{2+} / \mathrm{Ru}^{0}}$ to $0.0 \mathrm{~V}$. Directly after the potential step small $\mathrm{Ru}$ monolayer islands with diameters $\leq 20 \AA$ appear (examples marked by arrows). These Ru islands are found almost exclusively in the wider domains between the double rows of the reconstruction, i.e., in the regions where the $\mathrm{Au}$ atoms in the topmost layer occupy fcc sites. Although the number of elbows is low on this surface, neither these nor $U$ terminations of dislocation line pairs act as preferred nucleation sites, as was found, e.g., for $\mathrm{Ni} / \mathrm{Au}(111)[1,2,6]$. On partly unreconstructed surfaces (not shown) similar islands were observed in the unreconstructed areas. Since the latter areas also exhibit fcc stacking [9-11], we conclude that fcc domains represent preferred sites for $\mathrm{Ru}$ adisland nucleation.

With increasing $\mathrm{Ru}$ coverage the selective $\mathrm{Ru}$ nucleation in the fcc areas leads to the formation of an ordered

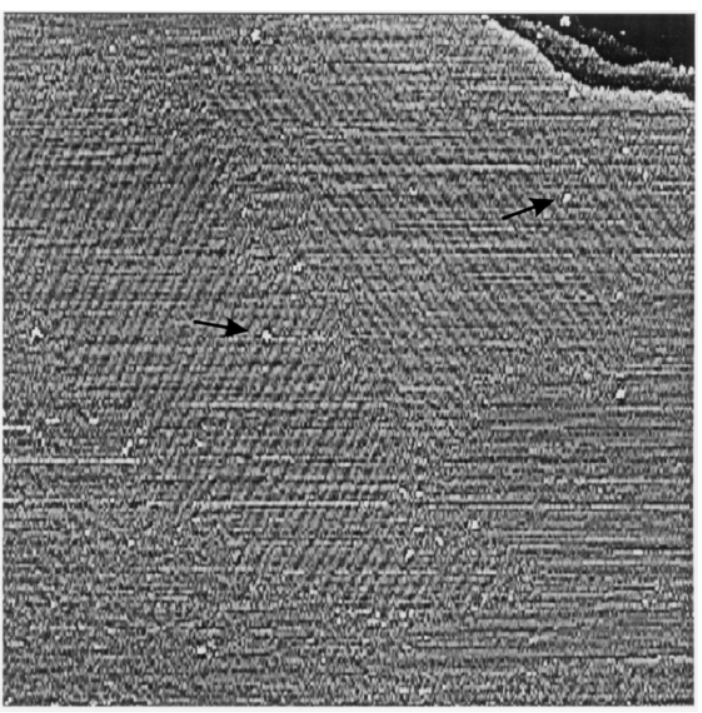

FIG. 1. STM image of $\mathrm{Au}(111)$ in $0.5 \mathrm{M} \mathrm{H}_{2} \mathrm{SO}_{4}+10^{-5} \mathrm{M}$ $\mathrm{RuCl}_{3}$, showing the predominant nucleation of the first $\mathrm{Ru}$ islands (e.g., at arrows) in the fcc areas of the Au reconstruction directly after a potential step from 0.15 to $0.0 \mathrm{~V}(1000 \times$ $1000 \AA^{2}$ ).

nanometer scale deposit pattern. Figure 2(a) shows the morphology after deposition of $0.07 \mathrm{ML}$ Ru. Although the coverage is still too low to produce a distinct, clearly visible pattern, it is obvious that the $\mathrm{Ru}$ adislands are neither randomly arranged on the surface nor nucleate at elbow sites or $\mathrm{U}$ terminations, but are predominantly

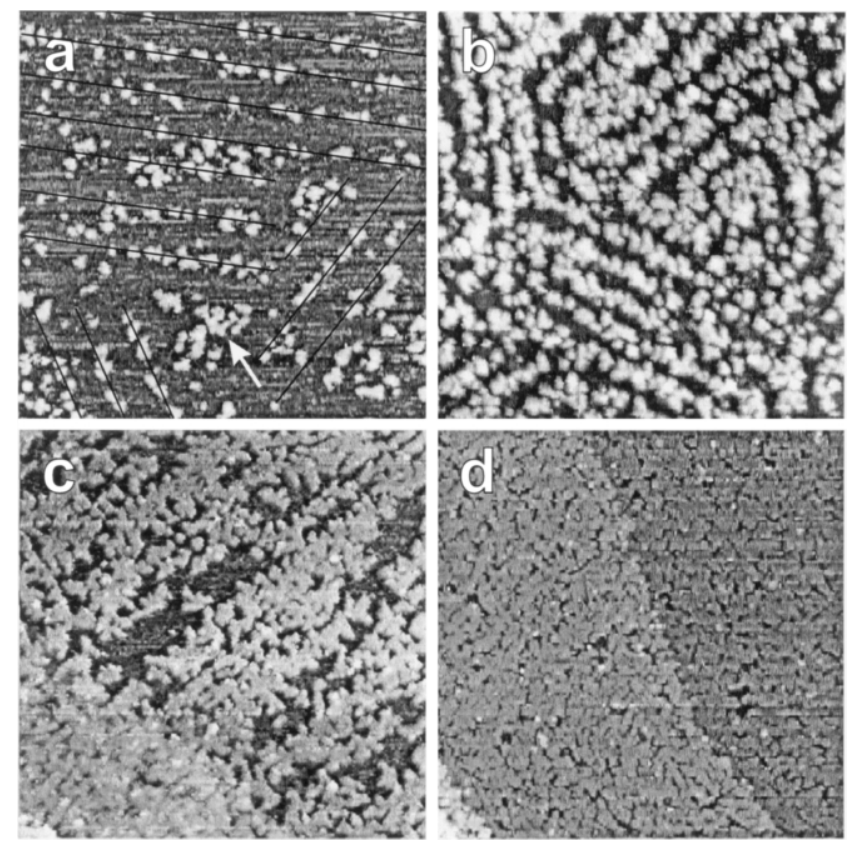

FIG. 2. STM images recorded on $\mathrm{Au}(111)$ in $0.5 \mathrm{M} \mathrm{H}_{2} \mathrm{SO}_{4}$ at $0 \mathrm{~V}$ after deposition of (a) $0.07 \mathrm{ML}\left(820 \times 820 \AA^{2}\right)$, (b) $0.30 \mathrm{ML}\left(820 \times 820 \AA^{2}\right)$, (c) $0.55 \mathrm{ML}\left(730 \times 730 \AA^{2}\right)$, and (d) $0.70 \mathrm{ML}\left(820 \times 820 \AA^{2}\right)$ of $\mathrm{Ru}$ from $0.5 \mathrm{M} \mathrm{H}_{2} \mathrm{SO}_{4}+$ $10^{-4} \mathrm{M} \mathrm{RuCl}_{3}$ at $0.0 \mathrm{~V}$. 
located along lines (black lines), which are separated by about $60 \AA$, equivalent to the unit cell spacing of the reconstructed surface $(63 \AA)$ and which exist in three directions rotated by $120^{\circ}$ with respect to each other. (The double row pattern of the reconstruction is not resolved in this image.) A similar adisland morphology is expected for the selective decoration of the fcc areas of the reconstructed surface by $\mathrm{Ru}$ islands. The occurrence of small areas with a more random arrangement of the adislands [example marked by white arrow in Fig. 2(a)] can be rationalized by defects in the reconstruction pattern and by unreconstructed areas, which are frequently observed at the boundaries of the reconstruction domains. For more quantitative data on the site selectivity the positions of 220 islands in the center of a single, extended domain were analyzed (for $\theta_{\mathrm{Ru}}=0.07 \mathrm{ML}$ ). Based on this analysis, less than $3 \%$ of the islands nucleated in the hcp areas. In addition, an average island size of $15 \AA$ and a typical next-neighbor island spacing along the rows of about $25 \AA$ [16] were obtained. From the latter a saturation island density of $6 \times 10^{12} \mathrm{~cm}^{-2}$ can be calculated (for a perfectly reconstructed surface).

With further increase in coverage the ordered pattern of the $\mathrm{Ru}$ adislands becomes more and more apparent. After deposition of $0.3 \mathrm{ML} \mathrm{Ru}$, the islands form strings of small $\mathrm{Ru}$ islands, which duplicate the fcc areas of the (previously) reconstructed surface [Fig. 2(b)]. At this coverage the saturation island density is reached. In fact, lateral growth of the islands leads already to beginning coalescence within the strings, finally resulting in nanowires of connected Ru adislands. At even higher coverages $(0.5 \mathrm{ML})$ coalescence begins also between islands of neighboring strings [Fig. 2(c)], leading to a percolation network of interconnected islands. Finally, at coverages close to a monolayer the surface is covered by a uniform, but highly defective Ru layer, in which the (formerly) fcc and hcp areas are almost indistinguishable [Fig. 2(d)].

The high saturation density of Ru monolayer islands, the irregular (ramified) shape of the larger, interconnected islands, and the high defect density at higher coverages are most simply explained by a very low mobility of the deposited $\mathrm{Ru}$ adatoms on the Au substrate and along the islands steps. In that case, however, one would expect preferential nucleation at the outer region of the fcc areas, which was not observed. An alternative explanation, which would account for the latter observation, involves an intrinsic limitation of the island size, presumably by strain effects. In that case higher adatom mobilities on the fcc areas were possible as well. A similar mechanism was suggested recently for $\mathrm{Ru}$ electrodeposition on $\mathrm{Pt}(111)$, where STM images showed small Ru monolayer islands of 20-50 Å average diameter at all deposition potentials [17].

In contrast to the well ordered $\mathrm{Ru}$ pattern observed upon deposition on the reconstructed $\mathrm{Au}$ substrate $\mathrm{Ru}$ deposition on the unreconstructed Au surface proceeds via homogeneous nucleation of $\mathrm{Ru}$ monolayer islands. This can be seen in the STM image in Fig. 3(a), showing the surface morphology after $\mathrm{Ru}$ deposition at $0.0 \mathrm{~V}$ on a $\mathrm{Au}(111)$ surface, where the reconstruction had previously been lifted by a potential excursion to more positive potentials. The island saturation density and the average island size, on the other side, are practically not affected by the structural change of the substrate and stay close to those obtained locally in the fcc areas of the reconstructed surface (for equal deposition times and Ru coverages). Similar results were obtained also for deposition at higher potentials, positive of the stability range of the $\mathrm{Au}(111)$ reconstruction [13].

Finally, homogeneous nucleation with an apparently random island distribution is also found if $\mathrm{Ru}$ is deposited on the reconstructed substrate at potentials $\leq-0.1 \mathrm{~V}$, i.e., at considerably higher deposition rate [see Fig. 3(b)]. Hence, the selective nucleation in fcc areas is restricted to low deposition overpotentials. This is in good agreement with observations of the initial stages of growth during $\mathrm{Ni}$ electrodeposition on $\mathrm{Au}(111)$ [2] and $\mathrm{Au}(100)$ [18], where selective nucleation at preferred sites was also restricted to low overpotentials, and with expectations from simple nucleation and growth theory, according to which the preference to inhomogeneous nucleation should decrease with increasing supersaturation.

In a microscopic picture the selective decoration of the fcc areas might be explained by a model similar to that originally proposed for $\mathrm{Ni}$ on reconstructed $\mathrm{Au}(111)$, where the selective nucleation of $\mathrm{Ni}$ islands at the elbow sites was attributed to a heterogeneity in the adsorption energy or diffusion barrier for $\mathrm{Ni}$ adatoms [1]. In an analogous way it may be supposed for $\mathrm{Ru}$ on $\mathrm{Au}(111)$ that the binding energy and/or diffusion barrier for Ru adatoms are enhanced in the fcc areas, resulting in local trapping and subsequent nucleation of $\mathrm{Ru}$ islands at these sites. A similar model was recently suggested to explain the local decoration of unreconstructed areas on a partly reconstructed $\mathrm{Au}(100)$ electrode surface by electrodeposited $\mathrm{Ni}$ adislands [18]. In that case the mobility of the Ru adatoms must be sufficiently high to allow for an effective transport of $\mathrm{Ru}$

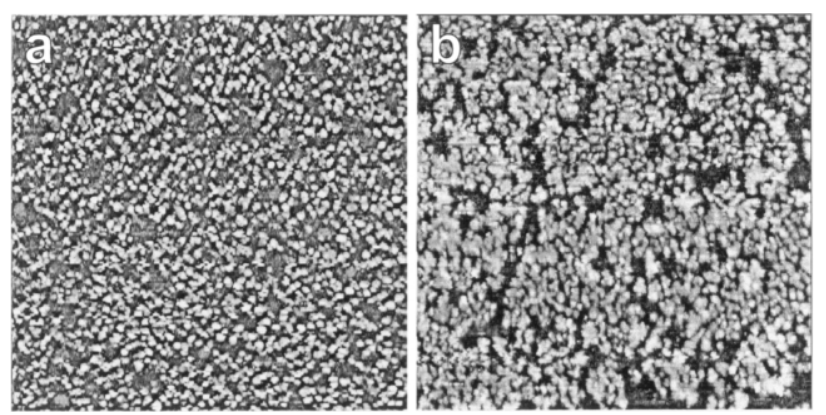

FIG. 3. STM images recorded on $\mathrm{Au}(111)$ in $0.5 \mathrm{M} \mathrm{H}_{2} \mathrm{SO}_{4}$ at $0.0 \mathrm{~V}$ after $\mathrm{Ru}$ deposition in $0.5 \mathrm{M} \mathrm{H}_{2} \mathrm{SO}_{4}+10^{-4} \mathrm{M} \mathrm{RuCl}_{3}$ (a) on an unreconstructed $\mathrm{Au}$ substrate at $0.0 \mathrm{~V}$ (0.20 ML; $1600 \times 1600 \AA^{2}$ ) and (b) on reconstructed $\mathrm{Au}(111)$ at $-0.2 \mathrm{~V}$, i.e., at higher overpotentials $\left(0.50 \mathrm{ML} ; 1200 \times 1200 \AA^{2}\right)$. 


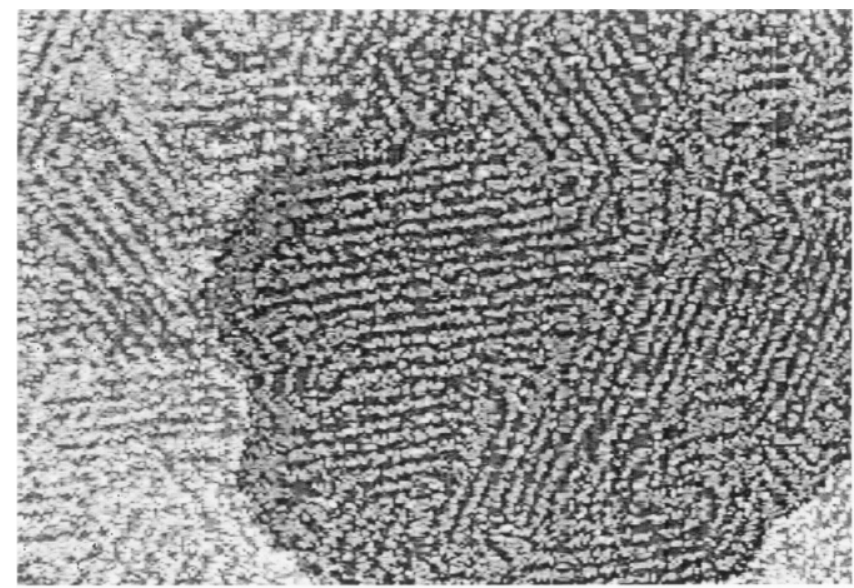

FIG. 4. STM image recorded on $\mathrm{Au}(111)$ in $0.5 \mathrm{M} \mathrm{H}_{2} \mathrm{SO}_{4}$ at $0.0 \mathrm{~V}$ after deposition of $0.30 \mathrm{ML}$ from $0.5 \mathrm{M} \mathrm{H}_{2} \mathrm{SO}_{4}+$ $10^{-4} \mathrm{M} \mathrm{RuCl}_{3}$ at $0.0 \mathrm{~V}\left(3200 \times 2200 \AA^{2}\right)$.

from the hcp into the fcc areas, i.e., over lengths on the order of (at least) the distance from the center of the hcp domains to the edge of the fcc domains (20-27 $\AA$ ). The high island density, however, could also emerge at much higher adatom mobilities if the growth of the islands is limited by other phenomena, such as lattice strain in the deposit. Alternatively, one could envision a mechanism involving place exchange of Ru adatoms with Au surface atoms and subsequent $\mathrm{Ru}$ adisland formation on top of the substitutional $\mathrm{Ru}$ atoms, similar to the mechanism recently recognized to be responsible for the site-selective growth of $\mathrm{Ni}$ on $\mathrm{Au}(111)$ [6]. It would be hard to understand, however, why place exchange should be preferred at the highly coordinated fcc sites, as compared to the less strongly bound $\mathrm{Au}$ surface atoms in the elbows of the dislocations, which are preferential exchange sites for $\mathrm{Ni} / \mathrm{Au}(111)$. Consequently, a mechanism involving place exchange as the initial nucleation step appears unlikely in the present case.

Finally, the high selectivity for nucleation and growth in fcc areas at $0.0 \mathrm{~V}$ affords the preparation of extended, periodic Ru adisland structures with well-defined, periodic spacings on the nm scale ("nanowire gratings"). The high perfection with which the $\mathrm{Au}(111)$ reconstruction pattern can be replicated is demonstrated in the large scale image in Fig. 4. The entire visible surface area exhibits a rowlike adisland pattern with uniform island densities, even in the vicinity of steps and a screw dislocation (upper left part of Fig. 4). The regular arrangement of the adstructures on a mesoscopic scale may result in interesting, novel properties of these bimetallic $\mathrm{Ru} / \mathrm{Au}(111)$ surfaces, for instance, regarding the catalytic activity.

In summary, we have presented in situ STM results on the electrodeposition of $\mathrm{Ru}$ on reconstructed $\mathrm{Au}(111)$ electrodes, which show selective nucleation of $\mathrm{Ru}$ adislands in the fcc areas of the reconstructed surface at low overpotentials. This allows the preparation of $\mathrm{Ru}$ adstructures with defined dimensions on the nm scale.
We gratefully acknowledge a fellowship by the Alexander-von-Humboldt foundation (S.S.), as well as financial support (O. M. M.) by the Deutsche Forschungsgemeinschaft.

*Present address: ITCM-Institute of Electrochemistry, University of Belgrade, P.O. Box 815, 11001 Belgrade, Yugoslavia.

[1] D. D. Chambliss, R. J. Wilson, and S. Chiang, Phys. Rev. Lett. 66, 1721 (1991).

[2] F. Möller, O. M. Magnussen, and R. J. Behm, Phys. Rev. Lett. 77, 5249 (1996).

[3] J. A. Meyer, P. Schmid, and R. J. Behm, Phys. Rev. Lett. 74, 3864 (1995).

[4] H. Brune, M. Giovanni, K. Bromann, and K. Kern, Nature (London) 394, 451 (1998); H. Brune, Surf. Sci. Rep. 31, 121 (1998); B. Fischer, H. Brune, J. V. Barth, A. Fricke, and K. Kern, Phys. Rev. Lett. 82, 1732 (1999).

[5] C. Günther, S. Günther, E. Kopatzki, R. Q. Hwang, J. Schröder, J. Vrijmoeth, and R. J. Behm, Ber. BunsenGes. Phys. Chem. 97, 522 (1993); C. Günther, J. Vrijmoeth, R. Q. Hwang, and R. J. Behm, Phys. Rev. Lett. 74, 754 (1995).

[6] J. A. Meyer, I. D. Baikie, E. Kopatzki, and R. J. Behm, Surf. Sci. 365, L647 (1996).

[7] J. V. Barth, H. Brune, G. Ertl, and R. J. Behm, Phys. Rev. B 42, 9307 (1990).

[8] D. M. Kolb and J. Schneider, Electrochim. Acta 31, 929 (1986).

[9] J. Wang, A. J. Davenport, H. S. Isaacs, and B. M. Ocko, Science 255, 1416 (1991).

[10] N. J. Tao and S. M. Lindsay, Surf. Sci. 274, L546 (1992).

[11] D. M. Kolb, Prog. Surf. Sci. 51, 109 (1996).

[12] J. Wiechers, T. Twomey, D. M. Kolb, and R. J. Behm, J. Electroanal. Chem. 248, 451 (1988).

[13] S. Strbac, O.M. Magnussen, and R.J. Behm (unpublished).

[14] K. Herrero, K. Franaszczuk, and A. Wieckowski, J. Electroanal. Chem. 361, 269 (1993).

[15] This type of topography is often observed on $\mathrm{Au}(111)$, in particular on flame annealed samples [10,11]. Although the origin of this behavior is not completely understood, it is most likely related to the annealing (temperature, annealing time) and/or immersion procedure.

[16] At island densities below the saturation value the islands are often not distributed statistically but for unknown reasons cluster in certain areas of the fcc areas. For this reason the typical island distance (i.e., the maximum in the nearest-neighbor distribution function) is significantly smaller than the average island distance ( $39 \AA$ for the analyzed sample).

[17] K. A. Friedrich, K.-P. Geyzers, A. Marmann, U. Stimming, and R. Vogel, Z. Phys. Chem. 208, 137 (1998); K. A. Friedrich, K.-P. Geyzers, U. Stimming, J. Stumper, and R. Vogel, Electrochem. Soc. Proc. Vol. 95-23, 299 (1995).

[18] F. A. Möller, O. M. Magnussen, and R. J. Behm, Z. Phys. Chem. 208, 57 (1998). 